\title{
The current status of the family Russulaceae in the Uttarakhand Himalaya, India
}

\author{
Joshi $\mathbf{S}^{*}$, Bhatt $\mathbf{R P}^{1}$, and Stephenson $\mathrm{SL}^{2}$ \\ ${ }^{1}$ Departmet of Botany and Microbiology, H. N. B. Garhwal University, Srinagar Garhwal, Uttarakhand 246 174, India - \\ swetajosh83@gmail.com, bhatt.rajendra123@gmail.com \\ ${ }^{2}$ Department of Biological Sciences, University of Arkansas, Fayetteville, Arkansas 72701, USA - slsteph@uark.edu
}

Joshi S, Bhatt RP, Stephenson SL 2012 - The current status of the family Russulaceae in the Uttarakhand Himalaya, India. Mycosphere 3(4), 486-501, Doi 10.5943 /mycosphere/3/4/12

The checklist provided herein represents a current assessment of what is known about the ectomycorrizal family Russulaceae from the Uttarakhand Himalaya. The checklist includes 105 taxa, 55 of which belong to the genus Lactarius Pers. ex S.F. Gray and 50 are members of the genus Russula Pers. ex S.F. Gray. Eleven of the species of Lactarius (listed as Lactarius sp. 1 to 11 in the checklist) are apparently new to science and have yet to be formally described.

Key words - Ectomycorrhizal fungi - Himalayan Mountains - Lactarius - Russula - Taxonomy

\section{Article Information}

Received 24 July 2012

Accepted 31 July 2012

Published online 28 August 2012

*Corresponding author: Sweta Joshi - e-mail - swetajoshi83@ gmail.com

\section{Introduction}

The family Russulaceae is one of the largest ectomycorrhizal families in the order Agaricales. The family was established by Roze (1876) as the Russulariees (non. nud.) to include the two agaric genera Russula and Lactarius, both of which were described originally by Persoon $(1796,1797)$. The members of the Russulaceae are characterized by their fleshy and often brightly coloured fruiting bodies with prominent lamellae, a heteromerous context traversed by conducting hyphae, the absence of clamp connections in almost all of the known taxa and warted, lightcolored amyloid spores that exhibit various types of exosporial ornamentation.

The northwestern subtropical and temperate forests of the Uttarakhand Himalaya are a major terrestrial carbon sink and represent one of the most threatened life support systems on earth. Having some knowledge of macrofungal diversity at the community and species level in these forests is an essential prerequisite for developing and understanding their overall ecology as well as assessing the crucial niche relationships of the various elements that make up the forest biota, including the macrofungi themselves. The large gap that exists with respect to our knowledge of macrofungal diversity in the Uttarakhand Himalaya is currently a serious constraint for being able to develop any type of understanding. For proper planning and management of these forests and the conservation of their biodiversity, characterization of both the systematics and ecology of the assemblage of macrofungi present is essential. This paper represents a step towards developing a comprehensive database of macrofungal diversity in India, specifically the status of the family Russulaceae in the Himalayas. We hope that it can serve as a starting point for future studies.

\section{Methods}

All available publications possibly containing one or more records of members of the Russulaceae reported from the Uttarakhand Himalaya were examined. All of these records were compiled and the nomenclature used 
for each species listed checked against the classification system provided on the Index Fungorum web site (http://www.indexfungorum.org/names/names. asp) and the nomenclature updated when necessary. The checklist compiled as a result of this effort is organized in alphabetical order. The name of the collecting locality and reference to the collector and/or source of the record are provided for each taxon. The 11 apparently new taxa in the genus Lactarius collected by the first two authors during studies carried out during the period of 2006 to 2009 are also included in the list.

It should be noted that the name used for the state of Uttarakhand in the checklist reflects changes that have occurred in the name of the state. Until 2000, it was part of the larger state known as Uttar Pradesh. However, in that year a portion of Uttar Prahesh was separated off to become the state of Uttaranchal. The name of the state was officially changed from its interim name Uttaranchal to Uttarakhand in 2007.

\section{Study area}

The Himalayas are one of the youngest folded mountainous formations in the world, and the youngest mountains in India. Uttarakhand is one of the hotspots of global biodiversity and is situated in the western part of the central Himalayas (Fig. 1). The unusually wide range of elevations, the rapid changes in environmental conditions that take place over even small distances and a high level of endemism make the region interesting for all types of studies. Uttarakhand enjoys one of the most important locations on the political map of India, extending from 28'43" $\mathrm{N}$ to 31 '28" N latitude and 77'34" $\mathrm{E}$ to 81 '03" $\mathrm{E}$ longitude. The total geographical area of the region is $53,483 \mathrm{~km}^{2}$ which accounts for nearly 15.5 per cent of the total geographical area of the western Himalayas and 1.63 per cent of the total land area of India. The recorded forest area of the state is approximately $34,650 \mathrm{~km}^{2}$ (Botanical Survey of India). Uttarakhand encompasses two distinct traditional politicocultural regions, the first known as Garhwal and the second as Kumaon. The state consists of 13 districts. These are Chamoli, Pauri, Tehri, Uttarkashi, Dehradun, Haridwar and
Rudraprayag in the Garhwal region and Nainital, Almora, Pithoragarh, Udham Singh Nagar, Champawat in Kumaon region. The state shares an international boundary with China in the north and Nepal in the east. Two of the largest and most importnat rivers in India, the Ganges and the Yamuna, originate in the glaciers of Uttarakhand. Uttarakhand is located east of the Yamuna River and actually serves as an intermediate zone between the western and central Himalayas in almost all respects, including the diversity of species. The state can be divided into three physiographic regions - the Himalayas, the Shivaliks and the lower elevation plains. The state has a temperate climate except for the plains, which have a subtropical type of climate. The climate of the state is influenced by the monsoon rhythm and is characterized by a precipitation regime different from that of the states of Jammu \& Kashmir and Himachal Pradesh to the west. The variable topography, soils, vegetation types and elevation are distinctive features of the state, resulting in a wide range of different microclimates from place to place. The high elevation regions of Uttarakhand Himalayas can be divided into three main climatic zones - the alpine zone, the temperate zone and the sub-tropical zone.

Six major forest types have been described in Uttarakhand. These are tropical moist deciduous forest, tropical dry deciduous forest, subtropical pine forest, Himalayan moist temperate forest, Himalayan dry temperate forest and subalpine/alpine forest (Champions $\&$ Seth 1968). The forest cover of the Uttarakhand Himalaya extends over an elevation range of 250 to $3500 \mathrm{~m}$ and most of the forests present are dominated by species of Quercus, Rhododendron, Pinus, Cedrus, Cupressus, Abies, Picea, Shorea and Betula. These forests provide sufficient diversity in vegetation and the favorable environmental conditions to support the growth and development of fleshy macrofungi.

\section{Brief Review of the Russulaceae}

Most studies of the systematics of the family Russulaceae have been carried out in Europe and North America. Maire (1910), Peck (1872, 1884, 1907), Burlingham (1907a, 1907b, 1908, 1910a, 1910b, 1913, 1915, 1918a, 


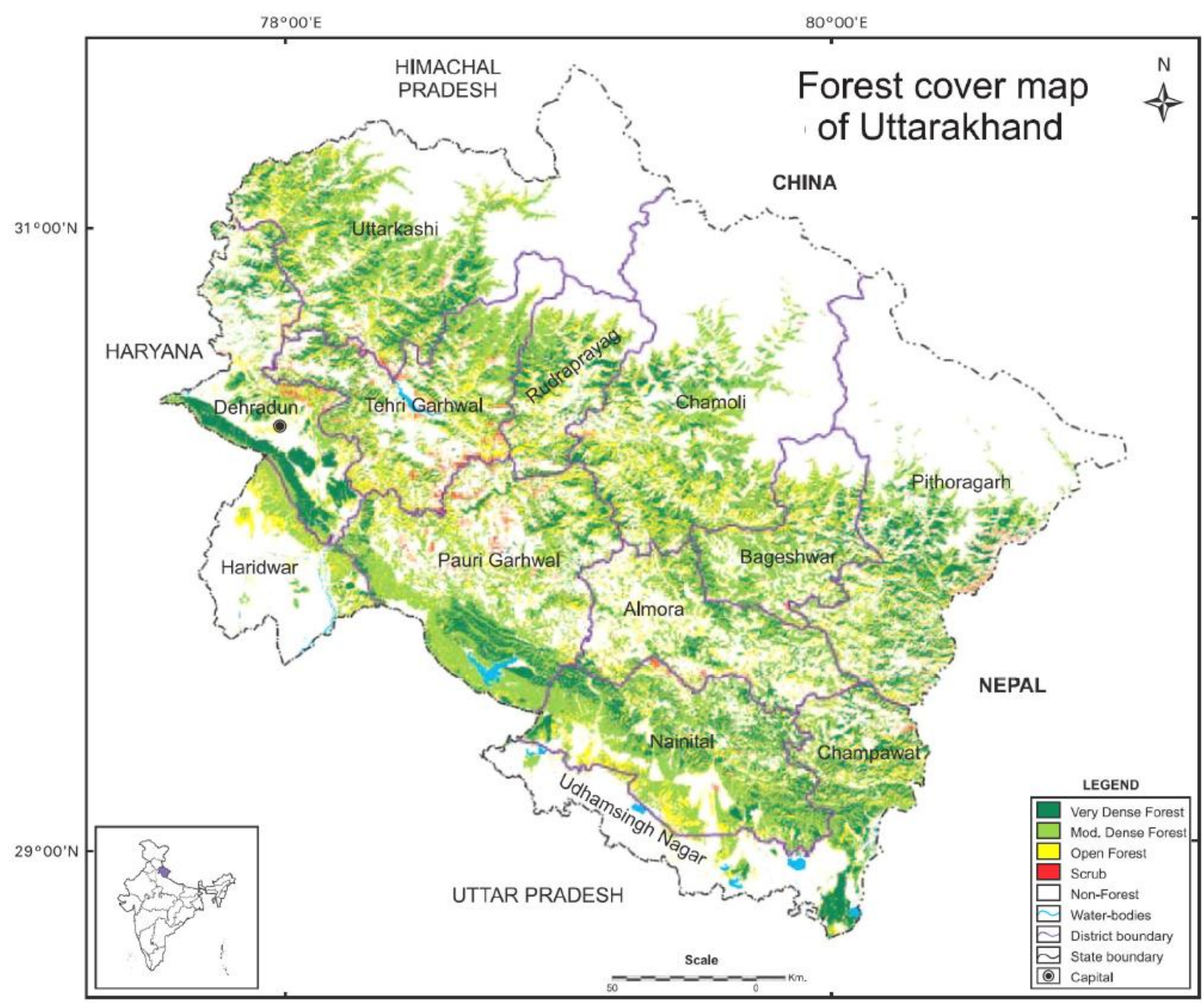

Fig. 1 - Map showing forest cover in the general study area.

1918b, 1921, 1924, 1932, 1940) and Beardslee (1918) were among the pioneer workers who initiated studies of the genera Lactarius and Russula. Knauth \& Neuhoff (1937), Neuhoff (1956), Pearson (1950), Hesler \& Smith (1960a, 1960b), Smith \& Hesler (1962), Blum (1962), Singer (1975), Hesler \& Smith (1979), Shaffer (1962, 1964, 1970a, 1970b, 1972) Schaeffer (1934), Romagnesi (1936, 1940, 1942, 1943, 1945, 1967, 1972), Singer (1926, 1928a, 1928b, 1986), Hesler (1960, 1961), Rayner (19681970), Buyck (1994, 1995, 1997), Kibby \& Fatto (1990) and Sarnari (1998) are some of the major monographic treatments of the family Russulaceae.

Verbeken (1998a), based on studies of 23 species of Lactarius from Africa, amended the subgenus Lactifluus of the genus Lactarius and excluded the temperate sections Albati (Bat.) Singer and Allardii Hesler \& Smith from the subgenus. Verbeken (1998b) also proposed amending the subgenus Lactariopsis (Henn.) R. Heim. to include the temperate section Albati (Bat.) Singer emend. Hesler \& A. H. Sm. She also discussed the relationships of the section Chamaeleontini with Russula subsection Amoeninae Singer ex Buyck and Lactariopsis with the genus Pleurogala. Verbeken \& Horak (2000) proposed 19 new taxa for the genus Lactarius and included three species described previously. They also presented a key to the species, illustrations and a discussion of their infrageneric position. Verbeken et al. (2000) recorded 35 species of Lactarius from relatively xeric Zambezian miombo woodlands in Zimbabwae. Miller \& Buyck (2002), Wang et al. (2006), Wang (2007), Nuytinck et al. (2006) and Buyck et al. (2007) studied the genus Lactarius in Madagascar. Huyen et al. (2007) and Buyck et al. (2008), who carried out molecular phylogenetic analyses of ribosomal genes (ITS-nuclear LSU) and part 
of a protein-coding gene (RPB2), indicated that four phylogenetically distinct clades could be identified within the family Russulaceae. Stubbe et al. (2010) studied species delimitation within the Lactarius gerardii species complex and attempted to define its taxonomic and geographical extent. Van de Putte et al. (2009, 2010), Stubbe et al. (2011) and Montoya et al. (2012) are others who have made recent contributions to what is known about the genus Lactarius. An outstanding monographic work, published by Sarnari (1998), considers European species of Russula. It deals with a critical analysis of all of the various nomenclatural problems and is probably the best currently available classification for the genus. Sarnari divided the genus into six subgenera (Amoenula, Compactae, Heterophyllidia, Icnrustatula, Ingratula and Russula) and stressed the importance of microscopic features. Adamcík et al. (2010) reported a new species of $R$. ochrifloridana from Florida, whereas other recent publications include those by Adamcík \& Buyck (2011), Buyck \& Adamcík (2011) and Buyck \& Atri (2011).

\section{Review of the Indian Literature}

The taxonomic study of macrofungi in India dates back to Linneaus, who in the $19^{\text {th }}$ century was the first person to name a species from India. Berkeley (1851, 1852, 1854, 1876) made a major contribution by reporting many agarics from India, including nine species of Russula and five species of Lactarius. The specimens upon which these records were based, had been collected from the Eastern and Western Himalaya by J.D Hooker. All of these are deposited in the herbarium of the Royal Botanic Gardens, Kew (K). More than a century later, Chona et al. (1958) reported Lactarius cillicioides from Delhi. More recent contributions to the study of macrofungi in India that have included some records of Lactarius include those of Bakshi (1974) and Patil \& Thite (1978) for western India; Sharma et al. (1978), Natarajan (1978), Kumar et al. (1979), Sathe \& Daniel (1980), Watling \& Gregory (1980) and Ray \& Samajpati (1980) for eastern India; Abraham et al. (1980, 1981, 1984), Abraham \& Kaul (1985) and Shajahan \& Samajpati (1995) for the eastern Himalayas; and Vrinda et al. 1997 for South India. Das et al. (2010) reported on the macrofungi of the Sikkim Himalaya. Dhancholia (2011) worked on family Russulaceae in Lahaul Valley, Himachal Pradesh.

Among the pioneer workers who made significant contributions to the study of the family Russulaceae in the northwestern Himalayas were Saini \& Atri (1981, 1982a, b, 1984, 1989a, b, 1990, 1993), Atri \& Saini (1986, 1988, 1989, 1990a, b, c), Saini et al. (1988, 1989), Atri et al. (1991a, b, c, d, 1992, 1993a, b, 1997), Rawla \& Sarwal (1983), Rawla (1994, 2001, 2002), Sarwal (1984), Lakhanpal et al. (1987), Lakhanpal (1993), Bhatt \& Lakhanpal (1988a, b, c, 1990), Bhatt et al. (1995, 1999, 2000), Das \& Sharma (2001a, b, 2002b, 2003, 2004, 2005a, b), Sharma et al. (2005), Sharma \& Das (2002, 2003), Das et al. (2002c, 2003b, 2004, 2005a, b, c, 2006a, b), Bhatt et al. (2007) and Dar et al. (2009).

Atri \& Saini (1989) reviewed the work carried out on the taxonomy of the family Russulaceae in India, whereas Atri et al. (1994) catalogued 48 taxa in the genus Lactarius and 67 taxa in the genus Russula described from India in the form of a checklist. Manjula (1983) revised the agaricoid and boletoid basidiomycetes reported from India and provided a modern checklist along with keys to the species. Atri et al. (1997) described the taxonomy, distribution, ecology and edibility of members of the genus Russula in the northwestern Himalayas and listed 74 taxa. Das \& Sharma (2002) provided a checklist of the genus Lactarius that included 56 species and 12 varieties described from India.

\section{Taxonomy}

The checklist that follows includes 105 taxa in the family Russulaceae recorded previously from the state of Uttarakhand. This total includes 95 species and 10 varieties. As mentioned earlier, Lactarius sp. 1 to 11 are apparently new and undescribed taxa represented by specimens collected in the Garhwal Himalaya. Most of the records in the checklist are based on material collected from forests of the temperate belt, within a range of elevations extending from 800 to $2300 \mathrm{~m}$. Most of the taxa involved were associated with 
various species of Quercus and Pinus, but Lactarius sp. 6 and Lactarius sp. 7 are two new species collected from Shorea robusta forests in the lower subtropical belt of the Sivaliks.

\section{Lactarius Pers. ex S.F. Gray}

Lactarius abbotanus K. Das \& J.R. Sharma, Mycotaxon 88: 378 (2003)

Locality - Abbot Mount, Champawat (Kumaon), Uttaranchal (Sharma \& Das 2003).

Lactarius alnicola var. alnicola A.H. Sm., Brittonia 12: 319 (1960)

Locality - Deoban, Chakrata, Uttar Pradesh (Atri \& Saini 1986, 1988).

Lactarius camphorates (Bull.) Fr., Epicr. Syst. Mycol. (Upsaliae): 346 (1838) [1836-1838]

Locality - Bageshwar (Kumaon), Uttaranchal (Das \& Sharma 2002).

Lactarius capitatus K. Das, J.R. Sharma \& Montoya, Mycotaxon 90: 286 (2004)

Locality - Loharkhet Top, Bageshwar (Kumaon), Uttaranchal (Das et al. 2004b).

Lactarius corrugis Peck, Ann. Rep. N. Y. St. Mus. Nat. Hist. 32: 31 (1880) [1879]

Locality - Phedkhal (Garhwal), Uttar Pradesh (Bhatt et al. 1999).

Lactarius dafianus K. Das, J.R. Sharma \& Verbeken, Mycotaxon 88: 338 (2003)

Locality - Kannar, Pithoragarh (Kumaon), Uttaranchal (Das et al. 2003).

Lactarius deliciosus var. deliciosus (L.) Gray, Nat. Arr. Brit. Pl. 1: 624 (1821)

Locality - Jamnotri (Garhwal) (Saini \& Atri 1982b, 1984, Atri et al. 1991b), Deoban (Garhwal) (Saini \& Atri 1982b).

Lactarius dhakuranius K. Das, Basso \& J.R. Sharma, Mycotaxon 91: 3 (2005)

Locality - Dhakuri, Bageshwar (Kumaon), Uttaranchal (Das et al. 2005c).

Lactarius dwalienesis K. Das, J.R. Sharma \& Verbeken, Mycotaxon 88: 334 (2003)

Locality - Khati, Bageshwar (Kumaon), Uttaranchal (Das et al. 2003).
Lactarius gerardii var. subrubescens (A.H. Sm. \& Hesler) Hesler \& A.H. Sm., North American Species of Lactarius: 114 (1979)

Locality - Dafia Dhura, Pithoragarh (Kumaon), Uttaranchal (Das \& Sharma 2005a).

Lactarius himalayanus Rawla \& Sarwal [as 'himalayana'], Biblthca. Mycol. 91: 42 (1983) Locality - Mussoorie, Uttar Pradesh (Rawla \& Sarwal 1983).

Lactarius hygrophoroides var. hygrophoroides Berk. \& M.A. Curtis, Ann. Mag. Nat. Hist. Ser. 34: 293 (1859)

Locality - Devidhar (Chamoli Garhwal); Khirsu (Pauri Garhwal), Uttar Pradesh (Bhatt et al. 1999).

Lactarius hygrophoroides var. lavendulaceus Hesler \& A.H. Sm. [as 'lavedulaceus'], North American Species of Lactarius: 176 (1979)

Locality - Gudkhiakhal (Tehri

Garhwal); Akhori, Khirsu, Uttar Pradesh (Bhatt et al. 1999).

Lactarius hygrophoroides var. odoratus Hesler \& A.H. Sm., North American species of Lactarius:173 (1979)

Locality - Dandapani, Khirsu, (Garhwal), Uttar Pradesh (Bhatt et al. 1999).

Lactarus hygrophoroides var. rugatus (Kühner\& Romagn.) Hesler \& A.H. Sm., North American Species of Lactarius: 175 (1979)

Locality - Nagdeva-Jhandidhar (Pauri Garhwal), Uttar Pradesh (Bhatt et al. 1999).

Lactarius indigo var. indigo (Schwein.) Fr., Epicr. Syst. Mycol.: 341 (1838) [1836-1838]

Locality - Majharatal (Tehri Garhwal), Uttaranchal (Sharma \& Das 2002).

Lactarius lignyotus var. canadensis A.H. Sm. \& Hesler, Brittonia 14: 398 (1962)

Locality - Dhakuri (Bageshwar), Mayawati (Champawat) Kumaon, Uttaranchal (Das \& Sharma 2004).

Lactarius lignyotus var. lignyotus Fr., Nuovi Ann. Sci. Nat.: 25 (1857)

Locality - Dhakuri, Bageshwar (Kumaon), Uttaranchal (Das \& Sharma 2005a). 
Lactarius maitlyensis K. Das, J.R. Sharma \& Verbeken, Mycotaxon 88: 336 (2003) Locality - Maitly Top, Pithoragarh (Kumaon), Uttaranchal (Das et al. 2003).

Lactarius mayawatianus K. Das \& J.R. Sharma, Mycotaxon 88: 380 (2003)

Locality - Mayawati, Champawat; Furti, Almora, (Kumaon), Uttaranchal (Sharma \& Das 2003).

Lactarius montoyae K. Das \& J.R. Sharma, Mycotaxon 89(2): 291 (2004)

Locality - Dhakuri, Bageshwar (Kumaon), Uttaranchal (Das \& Sharma 2004).

Lactarius mukteswaricus K. Das, J.R. Sharma \& Montoya, Fung. Diversity 16: 26 (2004)

Locality - Mukteshwar, Nainital (Kumaon), Uttaranchal (Das et al. 2004a).

Lactarius paradoxus Beardslee \& Burl. [as 'Lactaria paradoxa'], Mycologia 32: 584 (1940)

Locality - Deoban, Chakrata (Garhwal), Uttar Pradesh (Atri \& Saini 1986).

Lactarius piperatus var. glaucescens (Crossl.) Hesler \& A.H. Sm., North American Species of Lactarius: 186 (1979)

Locality - Almora, Pithoragarh, Uttarakhand (Das \& Sharma 2002).

Lactarius piperatus var. piperatus (L.) Pers., Tent. Disp. Meth. Fung.: 64 (1797)

Locality - Pauri, Dehradun, (Garhwal); Pithoragarh (Kumaon), Uttaranchal (Das \& Sharma 2002).

Lactarius romagnesii Bon, Docums Mycol. 9 (No. 35): 39 (1979)

Locality - Dhakuri (Kumaon),

Uttaranchal (Sharma \& Das 2001).

Lactarius rubrifluus Gillet, Bull. Soc. Linn. Normandie, Sér. 24: 255 (1870)

Locality - Budakedar, Tehri (Garhwal), Uttaranchal (Sharma \& Das 2002).

Lactarius sanguifluus (Paulet) Fr., Epicr. Syst. Myc.: 341 (1838) [1836-1838]
Locality - Garhwal Himalaya, Uttaranchal (Bhatt et al. 2000).

Lactarius sanjappae K. Das, J.R. Sharma \& Montoya, Fung. Diversity 16: 24 (2004).

Locality - Mukteshwar, Nainital (Kumaon), Uttaranchal (Das et al. 2004a).

Lactarius scrobiculatus var. canadensis (A.H. Sm.) Hesler \& A.H. Sm., North American Species of Lactarius: 297 (1979)

Locality - Gobind Dham, enroute Badrinath (Garhwal), Uttar Pradesh (Atri \& Saini 1988).

Lactarius scrobiculatus var. scrobiculatus (Scop.) Fr., Epicr. Syst. Mycol.: 334 (1838) [1836-1838]

Locality - Jamnotri, Hanuman Chatti (Saini \& Atri 1982b, 1984); Chakrata, Deoban (Atri \& Saini 1988) Garhwal, Uttar Pradesh.

Lactarius serifluus (DC.) Fr., Epicr. Syst. Mycol.: 345 (1838) [1836-1838]

Locality - Chamoli Garhwal, Uttarakhand (Das \& Sharma 2002).

Lactarius subdulcis (Pers.) Gray, Nat. Arr. Brit. Pl. 1: 625 (1821)

Locality - Quinkaleshwar (Garhwal), Uttaranchal (Bhatt et al. 2000).

Lactarius subindigo Verbeken \& E. Horak, Aust. Syst. Bot. 13: 651 (2000)

Locality - Abbot Mount, Champawat; Mayawati, Almora (Kumaon), Uttaranchal (Sharma \& Das 2003).

Lactarius subisabellinus var. murrillianus (A.H. Sm. \& Hesler) Hesler \& A.H. Sm., North American Species of Lactarius: 122 (1979)

Locality - Oli Forest, Joshimath (Garhwal); Naini Peak, Nainital (Kumaon); Deoban, Kailana, Chakrata (Garhwal), Uttar Pradesh (Atri \& Saini 1986, Saini \& Atri 1990).

Lactarius subpurpureus Peck, Ann. Rep. N. Y. St. Mus. Nat. Hist. 29: 43(1878) [1876]

Locality - Deoban, Chakrata (Garhwal), Uttar Pradesh (Atri \& Saini 1986, Saini \& Atri 1990). 
Lactarius subvellereus var. subdistans Hesler \& A.H. Sm., North American Species of Lactarius: 203 (1979)

Locality - Mukteshwar, Nainital; Abbot Mount, Champawat; Sandev, Pithoragarh (Kumaon), Uttaranchal (Das \& Sharma 2005a).

Lactarius vellereus (Fr.) Fr., Epicr. Syst. Mycol.: 340 (1838) [1836-1838]

Locality - Lal Tibba, Mussoorie (Garhwal), Uttar Pradesh (Rawla 1994).

Lactarius verbekenae K. Das, J.R. Sharma \& Montoya, Fung. Diversity 16: 29 (2004)

Locality - Mukteshwar, Nainital; Lohaghat, Champawat (Kumaon Himalaya), Uttaranchal (Das et al. 2004a).

Lactarius volemus var. flavus Hesler \& A.H. Sm., North American Species of Lactarius (Ann Arbor): 165 (1979)

Locality - Enroute to waterfall, Chakrata (Garhwal), Uttar Pradesh (Atri \& Saini 1986, Saini \& Atri 1993).

Lactarius volemus var. volemus (Fr.) Fr., Epicr. Syst. Mycol.: 344 (1838) [1836-1838]

Locality - Chakrata (Saini \& Atri 1984) and Mussoorie, Lal Tibba (Rawla 1994) (Garhwal), Uttar Pradesh.

Lactarius yazooensis Hesler \& A.H. Sm., North American Species of Lactarius: 264 (1979)

Locality - Mussoorie, on the way to Mossy Falls (Garhwal), Uttar Pradesh (Atri \& Saini 1986, 1988).

Lactarius zonarius var. riparius Hesler \& A. H. Sm., North American Species of Lactarius: 252 (1979)

Locality - Pauri (Garhwal), Uttaranchal (Das \& Sharma 2002).

Lactarius zonarius var. zonarius (Bull.) Fr., Epicr. Syst. Mycol.: 336 (1838) [1836-1838]

Locality - Mussoorie, Dhobighat (Garhwal), Uttar Pradesh (Saini \& Atri 1982a, Atri \& Saini 1988).

\section{Lactarius sp. 1}

Locality - Khirsu (Pauri Garhwal),
Uttarakhand. Collected August 2007, GUH M25219. Found in association with Quercus leucotrichophora.

Lactarius sp. 2

Locality - Chandrabadni (Tehri Garhwal), Uttarakhand. Collected August 2009, GUH M- 25601. Found in association with Quercus leucotrichophora and Picea spp.

Lactarius sp. 3

Locality - Bugani (Pauri Garhwal), Uttarakhand. Collected August 2009, GUH M25607. Found in association with Pinus roxburghii.

\section{Lactarius sp. 4}

Locality - Adwani (Pauri Garhwal), Uttarakhand. Collected August 2007, GUH M25223; GUH M-25225. Found in association with Quercus leucotrichophora and Rhododendron aroboreum.

\section{Lactarius sp. 5}

Locality - Khirsu (Pauri Garhwal), Uttarkhand. Collected August 2006, GUH M25010; Adwani, August, 2006, GUH M-25026. Found in association with Lyonia ovalifolia, Myrica esculenta, Quercus leucotrichophora and Rhododendron arboreum.

Lactarius sp. 6

Locality - Nanda Ki Chowki (Dehradun), Uttarakhand. Collected September 2008, GUH M- 25568, September 2009, GUH M-25573. Found in association with Shorea robusta.

Lactarius sp. 7

Locality - Tapovan (Dehradun), Uttarakhand. September 2008, GUH M-25448. Found in association with Shorea robusta.

\section{Lactarius sp. 8}

Locality - Kansh Khet (Pauri Garhwal), Uttarakhand. Collected August 2006, GUH M25012. Found in association with Quercus leuchotrichophora.

\section{Lactarius sp. 9}

Locality - Gundiyat (Uttarkashi), Uttarakhand. Collected October 2009, GUH 
M-25683; Found in association with Cedrus deodara.

\section{Lactarius sp. 10}

Locality - Hanuman Mandir (Pauri Garhwal), Uttarakhand. Collected August 2007, GUH M- 25230. Found in association with Pinus roxbrghii and Quercus leuchotrichophora.

\section{Lactarius sp. 11}

Locality - Jabbar Khet (Mussorrie), Uttarakhand. Collected August 2009 GUH M25619. Found in association with Quercus leucotrichophora and Rhododendron arboreum.

\section{Genus Russula Pers. ex S.F.Gray}

Russula abbotensis K. Das \& J.R. Sharma, Russulaceae of Kumaon Himalaya: 176 (2005) Locality - Abbot Mount, Champawat, (Kumaon), Uttaranchal (Das \& Sharma 2005b).

Russula adusta (Pers.) Fr., Epicr. Syst. Mycol.: 350 (1838) [1836-1838].

Locality - Kailana, Chakrata (Garhwal) (Saini \& Atri 1984, Atri and Saini 1990b), Lohaghat; Mayawati; Jageshwar, Champawat (Kumaon), Uttaranchal (Das \& Sharma 2005b).

Russula alachuana Murrill, Mycologia 30: 362 (1938)

Locality - Kailana, Chakrata (Garhwal), Uttar Pradesh (Atri \& Saini 1986).

Russula albida Peck, Bull. N. Y. St. Mus. Nat. Hist. 1 (No. 2): 10 (1887)

Locality - Nagdev-Jhandidhar Forest, Khirsu Forest (Garhwal), Uttar Pradesh (Bhatt et al. 1995).

Russula albonigra (Krombh.) Fr., Hymenomyc. Eur.: 440 (1874)

Locality - Deoban, Chakrata (Garhwal), Uttar Pradesh (Saini \& Atri 1984, Atri \& Saini 1986, 1990a).

Russula amoenicolor Romagn., Bull. Mens. Soc. Linn. Lyon 31: 175 (1962) Locality - Jabbar Khet, Mussoorie (Garhwal), Uttar Pradesh (Rawla \& Sarwal
1983).

Russula amoenicolor var. ramgarhensis $\mathrm{K}$. Das, J.R. Sharma \& R.P. Bhatt, Russulaceae of Kumaon Himalaya: 127 (2005)

Locality - Ramgarh, Nainital (Kumaon), Uttaranchal (Das et al. 2005a).

Russula amoenolens Romagn., Contribution a 1'Etude de Quelques Aspergilles 21: 111 (1952)

Locality - Nagdev-Jhandidhar Forest, Khirsu (Garhwal), Uttar Pradesh (Bhatt et al. 1995).

Russula anatina Romagn., Russules d'Europe Afr. Nord: 306 (1967)

Locality - Dafia Dhura, Pithoragarh; Dhakuri, Bageshwar (Kumaon), Uttaranchal (Das \& Sharma 2003).

Russula appendiculata K. Das, S.L. Mill. \& J.R. Sharma, Mycotaxon 95: 210 (2006)

Locality - Gagar, Nainital (Kumaon), Uttaranchal (Das et al. 2006a).

Russula atropurpurea (Krombh.) Britzelm., Bot. Zbl. 54: 99 (1983)

Locality - Chakrata (Garhwal), Uttar Pradesh (Atri \& Saini 1986, Saini \& Atri 1989b).

Russula aurata Fr., Epicr. Syst. Mycol.: 360 (1838) [1836-1838]

Locality - Deoban, Chakrata (Garhwal), Uttar Pradesh (Atri \& Saini 1986, Saini \& Atri 1989b, Saini et al. 1989).

Russula aurora (Krombh.) Bres., Fung. Trident. 2: 93 (1892)

Locality - Chakrata, Mussoorie, Uttar Pradesh (Saini et al. 1989).

Russula brevipes Peck, Ann. Rep. N. Y. St. Mus. 43: 66 (1890)

Locality - Jageshwar, Almora; Mayawati, Champawat (Kumaon), Uttaranchal (Das \& Sharma,2005b).

Russula brevipes var. acrior Shaffer, Mycologia 56: 223 (1964)

Locality - Lohaghat, Mayawati, 
Champawat; Sandev, Pithoragarh (Kumaon), Uttaranchal (Das \& Sharma 2005b).

Russula compacta Frost, Ann. Rep. Reg. N. Y. St. Mus. 32: 32 (1879)

Locality - Abbot Mt., Sandev, Dafia

Dhura (Kumaon), Uttaranchal (Das \& Sharma 2005b).

Russula congoana Pat., Cryptog. Mycol. 30(3): 336 (1914).

Locality - Mussoorie, Uttar Pradesh (Sarwal 1984).

Russula consobrina (Fr.) Fr., Epicr. Syst. Mycol.: 359 (1838) [1836-1838]

Locality - Jamnotri, Hanumanchatti (Garhwal), Uttar Pradesh (Atri \& Saini 1986, 1990a).

Russula cynoxantha (Schaeff.) Fr., Monogr. Hymenomyc. Suec. 2(2): 194 (1863)

Locality - Chakrata, Kailana (Atri \& Saini 1986, 1990c), Joshimath, Oli Forest (Atri \& Saini 1990c), Ramgarh, Nainital; Lohaghat, Champawat; Dafia Dhura, Maitly, Pithoragarh (Das \& Sharma 2005b).

Russula dafianus K. Das \& J.R. Sharma, Russulaceae of Kumaon Himalaya: 166 (2005)

Locality - Dhakuri, Bageshwar; Dafia

Dhura; Pithoragarh (Kumaon), Uttaranchal (Das \& Sharma 2005b).

Russula decipiens (Singer) Bon, Docums Mycol. 15 (No. 60): 42 (1985)

Locality - Mukteshwar, Nainital (Kumaon), Uttaranchal (Das \& Sharma 2005b).

Russula decolorans (Fr.) Fr., Epicr. Syst. Mycol.: 361 (1838) [1836-1838]

Locality - Champawat, Mayawati; Mornoulla, Almora; Nainital, Ramgarh (Kumaon), Uttaranchal (Das \& Sharma 2003).

Russula delica Fr., Epicr. Syst. Mycol.: 350 (1838) (1836-1838)

Locality: Chakrata, Deoban; Nainital, Nainapeak (Kumaon), Uttar Pradesh (Atri \& Saini 1986).
Russula dhakuriana K. Das, J.R. Sharma \& S.L. Mill., Mycotaxon 95: 208 (2006)

Locality - Dhakuri, Bageshwar (Kumaon), Uttaranchal (Das et al. 2006a).

Russula dissimulans Shaffer, Brittonia 14: 267 (1962)

Locality - Adwani Forest (Garhwal), Uttar Pradesh (Bhatt et al. 1995).

Russula flavida var. dhakurianus K. Das, J.R. Sharma \& R.P. Bhatt, Russulaceae of Kumaon Himalaya: 203 (2005)

Locality - Dhakuri, Bageshwar (Kumaon), Uttaranchal (Das \& Sharma 2005a).

Russula flocculosa Burl., N. Amer. Fl. 9(4): 213 (1915)

Locality - Chobattakhal, Phaedkhal, Pauri Garhwal, Uttarakhand (Bhatt et al. 2007).

Russula foetens (Pers.) Pers., Observ. Mycol. 1: 102 (1796)

Locality - Joshimath, Nainital, Chakrata, Deoban, Jamnotri (Garhwal), Uttar Pradesh (Saini \& Atri 1984).

Russula himalayana Rawal \& Sarwal, Biblthca Mycol. 91: 28 (1983)

Locality - Jabbar Khet, Mussoorie (Garhwal), Uttar Pradesh (Rawla \& Sarwal 1983).

Russula mayawatiana K. Das, S.L. Mill. \& J.R. Sharma, Mycotaxon 95: 206 (2006)

Locality - Champawat, Mayawati (Kumaon), Uttaranchal (Das et al. 2006a).

Russula minutula var. minutula Velen., České Houby 1: 133 (1920)

Locality - Alumandi, Kailana (Saini \& Atri 1984, Saini et al. 1989); Mussoorie, enroute to Dhobighat; Joshimath (Saini et al. 1989) (Kumaon), Uttar Pradesh.

Russula mukteshwarica K. Das, S.L. Mill., J.R. Sharma \& R.P. Bhatt, Mycotaxon 94: 86 (2005)

Locality - Mukteshwar, Naintal; DafiaDhura, Pithoragarh; Nagdev Forest, Pauri 
(Das et al. 2005b).

Russula mussooriensis Rawla \& Sarwal, Biblthca Mycol. 91: 25 (1983)

Locality - Jabbar Khet, Mussoorie (Garhwal), Uttar Pradesh (Rawla \& Sarwal 1983).

Russula mustelina Fr., Epicr. Syst. Mycol.: 351 (1838) [1836-1838]

Locality - Oli Forest (Saini \& Atri 1984); Chakrata, Kailana (Atri \& Saini 1986, 1990c).

Russula natarjanii K. Das, J.R. Sharma \& Atri, Mycotaxon 95: 272 (2006)

Locality - Dhakuri, Bageshwar (Kumaon), Uttaranchal (Das et al. 2006b).

Russula nigricans Fr., Epicr. Syst. Mycol.: 350 (1838) [1836 -1838]

Locality - Chakrata, Uttar Pradesh (Saini \& Atri 1984, Saini et al. 1988).

Russula ochroleuca (Pers.) Fr., Epicr. Sys. Mycol.: 358 (1838) [1836 -1838]

Locality - Chakrata, Uttar Pradesh

(Saini et al. 1989).

Russula odorata Romagn., Bull. Mens. Soc. Linn. Lyon 19: 76 (1950)

Locality - Jabbar Khet, Mussoorie, Uttar Pradesh (Rawla \& Sarwal 1983).

Russula pectinata (Bull.) Fr., Fr. Epicr. Syst. Mycol.: 358 (1838) [1836-1838]

Locality - Jabbar Khet, Mussoorie, Uttar Pradesh (Rawla \& Sarwal 1983).

Russula praetervisa Sarnari, Mongrafia Illustrata del Genere Russula in Europe 1: 463 (1998)

Locality - Dhakuri, Bageshwar; Mayawati, Champawat; Mornoula, Champawat, Uttarakhand (Sharma et al. 2005).

Russula pseudolepida Singer, Bull. Trimest. Soc. Mycol. Fr. 55: 251 (1940) [1939]

Locality - Phedkhal, Pauri Garhwal; Mandal, Chamoli, Uttarakhand (Bhatt et al. 2007).
Russula puellaris var. atrii K. Das, S.L. Mill. \& J. R Sharma, Mycotaxon 95: 211 (2006)

Locality - Dhakuri, Bageshwar, Uttaranchal (Das et al. 2006a).

Russula pulverulenta Peck, Bull. Torrey Bot. Club 29: 70 (1902)

Locality - Teka, Gandera, Pauri Garhwal, Uttarakhand (Bhatt et al. 2007).

Russula raoultii Quél., Compt. Rend. Assoc. Franc. Avancem. Sci. 14(2): 449 (1886)

Locality - Dafia Dhura, Pithoragarh; Mornoulla, Almora, Kumaon, Uttaranchal (Das \& Sharma 2003).

Russula rhodomelanea Sarnari, Riv. Micol. 36: 53 (1993)

Locality - Dhakuri, Bageshwar, Uttaranchal (Das \& Sharma 2001b).

Russula schaefferina Rawla \& Sarwal, Biblthca Mycol. 91: 32 (1983)

Locality - Mussoorie, Uttar Pradesh (Rawla \& Sarwal 1983).

Russula silvicola Shaffer, Beih. Nova Hedwigia 51: 229 (1975)

Locality - Khirsu, Pauri Grahwal, Uttarakhand (Bhatt et al. 2007).

Russula subfoetens W.G. Sm., J. Bot., Lond. 11: 337 (1873)

Locality - Chakrata, Uttar Pradesh (Atri \& Saini 1986, Saini \& Atri 1989a).

Russula velenovskyi Melzer \& Zvára, Arch, Přirodov. Výzk. Čech. 17: 92 (1927)

Locality - Oli Forest, Joshimath, Uttar Pradesh (Atri \& Saini 1986, Saini \& Atri 1989b).

Russula virescens(Schaeff.) Fr., Anteckn. Sver, Ätl. Svamp.: 50 (1836)

Locality - Dhobighat, Mussoorie; Chakrata, Deoban, Uttar Pradesh (Saini et al. 1988).

\section{Acknowledgements}

This work was supported by a grant to Sweta Joshi from the University Grants 
Commission of India. The authors sincerely appreciate the assistance of Dr. Makan Prakash Vishwakarma and Rahul Mahar, H.N.B. Garhwal University, India for providing help during the sample collection from the Uttarakhand Himalaya.

\section{References}

Abraham SP, Kaul TN. 1985 - Larger fungi from Upsala Kashmir-111. Kavaka 12, $77-81$.

Abraham SP, Kachroo JL, Kaul TN. 1980 Fleshy fungi of Gulmarg Forest-1. Kavaka 8, 29-39.

Abraham SP, Kaul TN, Kachroo JL. 1981 Larger fungi from Kashmir-1. Kavaka 9, 35-43.

Abraham SP, Kaul TN, Kachroo JL. 1984 Larger fungi from Kashmir-II. Kavaka $12,41-48$.

Adamcík S, Buyck B. 2011 - The species of Russula subsection Xerampelinae described by C. H. Peck and Miss G. S. Burlingham. Cryptogamie Mycologie 32, 63-81.

Adamcík S, Mitchell D, Buyck B. 2010 Russula ochrifloridana, sp. nov., a new yellowish fishy Russula from Florida and its comparison with $R$. grundii. Cryptogamie Mycologie 31, 363-372.

Atri NS, Saini SS. 1988 - Studies on Lactarius Pers. - The subgenus Piperites (Fr.) Kauff. Kavaka 16, 13-19.

Atri NS, Saini, SS. 1986 - Further contributions on the studies of northwest Himalayan Russulaceae. Geobios New Reports 5, 100-105.

Atri NS, Saini SS. 1989 - Family Russulaceae Roze- a review. In: ML Trivedi, BS Gill, SS Saini (eds.). 115-128. Plant Science Research in India, Present Status and Future Challenges, New Delhi.

Atri NS, Saini SS. 1990a - Studies on Russula Pers. Section Decolorantes (Maire) Sing. Geobios New Reports 9, 10-13.

Atri NS, Saini SS. 1990b - North Indian Agaricales-VIII. The section compactae Fr. of Russula Pers. in India. Journal of the Indian Botanical Society 69, 343-346.
Atri NS, Saini SS. 1990c - North Indian Agaricales- X. Journal of the Indian Botanical Society 69, 425-429.

Atri NS, Saini SS, Mann, DK. 1991a - Further studies on North West Indian Agarics Systematics of Lactarius deliciosus (Fr.) S. F. Gray. Geobios New Reports 10, 106-111.

Atri NS, Saini SS, Mann DK. 1991b - Studies on north west Indian agarics: The genus Lactarius. Indian Phytopathology 44, 185-192.

Atri NS, Saini SS, Mann DK. 1991c - Further studies on North West Indian AgaricsSystematics of Lactarius deliciosus (Fr.) S. F. Gray. Geobios New Reports 10, 106-111.

Atri NS, Saini SS, Mann DK. 1991d - Genus Russula Pers in Dalhousie. In: NC Aery \& BL Chaudhary (eds.) 92-99. Botanical Researches in India, Udaipur.

Atri NS, Saini SS, Saini MK, Gupta AK. 1992 - Two new records of genus Russula from India. Geobios New Reports 11, 101-103.

Atri NS, Saini SS, Saini MK. 1993a - Some Russulaceous fungi from Dalhousie (H.P.) - The genus Lactarius Pers. Journal of the Indian Botanical Society $72,155-158$.

Atri NS, Saini SS, Saini MK, Gupta AK. $1993 b$ - Systematic studies on Russulaceous fungi — The genus Lactarius Pers. Journal of the Indian Botanical Society 72, 155-158.

Atri NS, Saini MK, Saini SS. 1994 - Indian Russulaceae Roze - A checklist, In TA Sharma, SS Saini, ML Trivedi and M Sharma (eds.) Current Researches in Plant Science. Dehradun.

Atri NS, Saini SS, Saini MK. 1993a - Some Russulaceous fungi from Dalhousie (H.P.) - The genus Russula Pers. Geobios New Reports 12, 137-140.

Atri NS, Saini SS, Saini MK. 1997 - Studies on genus Russula Pers. From north western Himalayas. Mushroom Research 6(1), 1-6.

Bakshi BK. 1974 - Mycorrhiza and its role in forestry. Forest Research Institute, Dehradun.

Beardslee CH. 1918 - The Russulas of North 
Carolina. J. Elisha Mitchell Sci. Soc. 33, 147-197.

Berkeley MJ. 1851 - Decades of fungi: Decades XXXII, XXXIII. Sikkim Himalayas fungi collected by Dr. Hooker. Hooker's Journal of Botany 3, 39-49.

Berkeley MJ. 1852 - Decades of Fungi XXXIX, XL Sikkim and Khassya and Khassya fungi. Hooker's Journal of Botany 4, 130-142.

Berkeley MJ. 1854 - Decades 41- 43. Indian fungi. Hooker's Journal of Botany 6, 129-143.

Berkeley MJ. 1876 - Three fungi from Kashmir. Grevillea 4, 137-138.

Bhatt RP, Lakhanpal TN. 1988a - Lactarius hygrophoroides Berk. \& Curt. An edible milky mushroom new to India. Current Science 57, 38-39.

Bhatt RP, Lakhanpal TN. 1988b - Russula crustosa Peck. An addition to Indian edible mushrooms. Current Science 57, 560-561.

Bhatt RP, Lakhanpal TN. 1988c - A new record of edible Russula from India. Current Science 57, 1257-1258.

Bhatt RP, Lakhanpal TN. 1990 - Fleshy fungi of north-western Himalayas-V. Indian Phytopathology 43, 156-164.

Bhatt RP, Bhatt VK, Gaur RD. 1995 - Fleshy fungi of Gahwal Himalaya: The genus Russula. Indian Phytopathology 48, 402-411.

Bhatt VK, Bhatt RP, Gaur RD. 1999 - Studies on the section Lactifluus, of the genus Lactarius in India. Indian Phytopathology 52(3), 236-244.

Bhatt VK, Bhatt RP, Gaur RD. 2000 Mushroom of the Garhwal Himalaya: The genus Lactarius Pers. ex. S. F. Gray. Mushroom Research 9, 11-18.

Bhatt RP, Purnima S, Semwal KC. 2007 - New records of Russula from Garhwal Himalaya. Mushroom Research 16, 5560.

Blum J. 1962 - Les Russules Paul Lechevalier, Paris.

Burlingham GS. 1907a - Some Lactarii from Windham County, Vermont. Bulletin of the Torrey Botanical Club 34, 85-95.

Burlingham GS. 1907b - Suggestions for the study of Lactariae. Torreya 7, 118-123. Burlingham GS. 1908 - A study of the Lactariae of the United States. Memoirs of the Torrey Botanical Club 14, 1-109.

Burlingham GS. 1910a - The Lactariae of North America Fasc. I and II. Mycologia 2, 27-36.

Burlingham GS. 1910b - Lactaria Pers. North American Flora 9, 172-200.

Burlingham GS. 1913 - The Lactariae of the Pacific Coast. Mycologia 2, 27-36.

Burlingham GS. 1915 - Russula Pers. North American Flora 9, 1-236.

Burlingham GS. 1918a - New species of Russula from Massachusetts. Mycologia 10, 93-96.

Burlingham GS. 1918b - A preliminary report of the Russulae of Long Island. Memoirs of the Torrey Botanical Club 17, 301-306.

Burlingham GS. 1921 - Some new species of Russula. Mycologia 13, 129-134.

Burlingham GS. 1924 - Notes on species of Russula. Mycologia 16, 129-134.

Burlingham GS. 1932 - Two new species of Lactaria. Mycologia 24, 460-463.

Burlingham GS. 1940 - Interesting species of Lactariae from Florida. Mycologia 40, 575-586.

Buyck B. 1994 - Flore illustree Des Champignons D'Afrique Centrole Russula II (Russulaceae), 411-539, fig. 258-351, p1. 69-87, Meise.

Buyck B. 1995 - A global and integrated approach on the taxonomy of Russulales. Russulales News 3, 3-17.

Buyck B. 1997 - Flore illustree Des champignons D'Afrique CentraleRussula III (Russulacea) 545-598, fig. 352-364. p1. 88-93, Meise.

Buyck B, Adamcík S. 2011 - Type studies of Russula species described by W. A. Murrill, $1 . \quad R$. roseiisabellina, $R$. sericella, and $R$. obscuriformis. Mycotax on 115, 131-144.

Buyck B, Atri NS. 2011 - A Russula (Basidiomycota, Russulales) with an unprecedented hymenophore configuration from NW Himalaya (India). Cryptogamie Mycologie 32, 185-190.

Buyck B, Verbeken A, Eberhardt U. 2007 - 
The genus Lactarius in Madagascar. Mycol. Res. 111, 787-798.

Buyck B, Hofsteller V, Eberhardt U, Verbeken A, Kauff F. 2008 - Walking the thin line between Russula and Lactarius: the dilemma of Russula subsection Ochricompactae. Fungal Diversity 28, 15-40.

Champions HG, Seth SK. 1968 - A revised survey of forest types of India. Govt of India publication, New Delhi $404 \mathrm{p}$.

Chona BL, Lall G, Kakria NC. 1958 - The fungi of Delhi. ICAR Bulletin 81, 43.

Dar GH, Beig MA, Ganai. 2009 - Hitherto unrecorded macrofungi from India. Applied Biological Research 11, 59-62.

Das K, Sharma JR. 2001a - A new record of Lactarius from India. Annals of Forestry 9, 284-286.

Das K, Sharma JR. 2001b - Russula rhodomelanea Sarnari - a new record for India. Mushroom Research 10, 109111.

Das K, Sharma JR. 2002 - The genus Lactarius in India. Bulletin Botanical Survey of India 44, 75-88.

Das K, Sharma JR. 2003 - New records of Russula from Kumaon Himalaya. Indian Journal of Forestry 26, 320-326.

Das K, Sharma JR. 2004 - Lactarius in Kumaon Himalaya 2. New and interesting species of subgenus Plinthogali. Mycotax on 89, 289-296.

Das K, Sharma JR. 2005a. New records of Lactarius from India. Annals of Forestry 13, 1-8.

Das K, Sharma JR. 2005b - Russulaceae of Kumaon Himalaya. Botanical Survey of India, Ministery of Environment and Forests, Govt. of India, Kolkata.

Das K, Sharma JR, Bhatt RP. 2002c - Russula flavida Frost - an addition to the Indian ectomycorrhizic fungi. Mushroom Research 11, 9-10.

Das K, Sharma JR, Verbeken A. 2003 - New species of Lactarius from Kumaon Himalaya, India. Mycotaxon 88, 333342.

Das K, Sharma JR, Montoya L. 2004a Lactarius in Kumaon Himalaya 1. New species of subgenus Russularia. Fungal Diversity 16, 23-33.
Das K, Sharma JR, Montoya L. 2004b Lactarius in Kumaon Himalaya 3. A new species of subgenus Lactifluus. Mycotaxon 90, $285-290$.

Das K, Sharma, JR, Bhatt RP. 2005a - Two new varieties of Russula from India. Annals of Forestry. 13, 281-286.

Das K, Miller SL, Sharma JR, Sharma P, Bhatt RP. 2005b - Russula in Himalaya 1: A new species of subgenus Amoneula. Mycotaxon 94, 85-88.

Das K, Sharma JR, Basso MT, Bhatt RP. 2005c - Lactarius in Kumaon Himalaya 4: A new species of subgenus Piperites. Mycotaxon 91, 1-7.

Das K, Miller SL, Sharma JR. 2006a Russula in Himalaya 2: Four new taxa. Mycotax on 95, 205-215.

Das K, Sharma JR, Atri NS. 2006b - Russula in Himalaya 3: A new species of subgenus Ingatula. Mycotaxon 95, 271-275.

Das K, Van de Putte K, Buyck B. 2010 - New or interesting Russula from Sikkim Himalaya (India). Cryptogamie Mycologie 31, 373-387.

Dhancholia S. 2011 - New records of genus Russula from dry temperate zone of Lahul Valley of Himachal Pradesh. Plant Disease Research 26, 203.

Hesler LR. 1960 - A study of Russula types-I. Memoirs of the Torrey Botanical Club 21, 1-59.

Hesler LR. 1961 - A Study of Russula type-II. Mycologia 53, 605- 625.

Hesler LR, Smith AH. 1960a - Studies on Lactarius-1. The North American species of section Lactarius. Brittonia 12, 119-139.

Hesler LR, Smith AH. 1960b - Studies on Lactarius-11. The North American species of section Scrobiculatus crocei Theiogali and Vellus. Brittonia 12, 306-350.

Hesler LR, Smith AH. 1979. North American Species of Lactarius. The University of Michigan Press, USA.

Huyen TH, Nuytinck J, Verbeken A, Lumyoung S, Desjardin D. 2007 Lactarius in Northern Thailand: 1. Lactarius subgenus Piperites. Fungal Diversity 24, 173-224. 
Kibby G, Fatto RM. 1990 - Keys to the species of Russula in northeastern North America, Kibby-fatto Enterprises USA.

Knauth B, Neuhoff W. 1937 - Die Milchlinge (Lactarii). In Die Wernerk (ed). Leipzig.

Kumar A, Lakhanpal TN, Stephenson SL. 1990 - Ecological studies of some macrofungi in the northwestern Himalayas. Nova Hedwigia 50, 535547.

Lakhanpal TN. 1993 - The Himalayan Agaricales - Status of systematics. Mushroom Research 2, 1-10.

Lakhanpal TN, Bhatt RP, Kaisth K. 1987 Lactarius sanguifluus Fr. An edible mushroom new to India. Current Science 56, 148-149.

Maire R. 1910 - Les bases de La classification dans le genre Russula. Bulletin de la Societe Mycogique de France 26, 1-77.

Manjula B. 1983 - A revised list of agaricoid and boletoid basidiomycetes from Nepal and India. Proceedings of the Indian Acadamey of Plant Sciences 92, 81-214.

Manoharachary C, Sridhar K, Singh K, Adholeya R, Suryanarayanan TS, Rawat S, Johri BN. 2005 - Fungal diversity: Distribution, conservation and prospecting of fungi from India. Current Science 89, 58-71.

Miller SL, Buyck B. 2002. - Molecular phylogeny of the genus Russula in Europe with a comparison of modern infrageneric classification. Mycological Research 106, 259-276.

Montoya L, Bandala, VM, Haug I, Stubbe D. 2012 - A new species of Lactarius (subgenus Gerardii) from two relict Fagus grandifolia var. mexicana population in Mexican montane cloud forests. Mycologia 104, 175-181.

Natarajan K. 1978 - South Indian AgaricalesV. Kavaka 6, 65-70.

Neuhoff W. 1956 - Die Miclinge: Pilze Mitteleuropas, Vol. II B, Bad Heilbrum.

Nuytinck J, Wang XH, Verbeken A. $2006-$ Description and taxonomy of the Asian representatives of Lactarius sect. Deliciosi. Fungal Diversity 22, 171203.
Patil MS, Thite AN. 1978 - Some fleshy fungi from Maharashtra-II. Botanique 9, 194 202.

Pearson AA. 1950 - The genus Lactarius. The Naturalist. University of Leeds, London.

Peck CH. 1872 - Genus Lactarius Fr. in Report of the Botanist for (1869). Annual Report New York State Cabinet. 23, 114-120.

Peck CH. 1884 -New York species of Lactarius. Reports of the State Botanist of New York 38, 111-133.

Peck CH. 1907 - New York species of Russula. NY State Museum Bulletin 116, 67-98.

Persoon CH. 1796 - Observationes mycologicae. Leipizig, Germany.

Persoon CH. 1797. Tentamen dispositionis methodica fungorum in classes ordines genera et familias cum suppl adjecta. Lipsiae.

Rawla GS. 1994 - Three species of Lactarius from Mussoorie Hills. Indian Science Congress Proceedings, Section VIII Bot. p 32.

Rawla GS. 2001 - Himalayan Species of Russula Pers ex S. F. Gray, In: PC Pande \& SS Samant (eds.). 1-48. Plant Diversity of the Himalaya. $626 \mathrm{pp}$.

Rawla GS. 2002 - Lactarius DC ex S. F. Gray in India-list and critical review, In: TPullaiah (ed.). 221-255. Biodiversity in India. 375 pp.

Rawla GS, Sarwal BM. 1983 - Taxonomic studies on Indian Agarics 1. Russulaceae. Bibliotheca Mycologica 91, 23-46.

Ray S, Samajpati N. 1980 - Agaricales of W. Bengal -VI. Indian Journal of Mycological Research 18, 87-96.

Rayner RW. 1968-1970 - Keys to the British species of Russula, 2nd ed. British Mycological Society Cambridge, London.

Romagnesi H. 1936 - Les Russules Supplement a La Revue de Mycologique 1, 3-14.

Romagnesi H. 1940 - Une nouvelle Russula du Grupe 'Integra'. Bulletin de la Societe Mycologique de France 56, 65-70.

Romagnesi H. 1942 - Contribution a'l'etude 
des Russules de la flora francaise-1, Bulletin de la Societe Mycologique de France 48, 150-169.

Romagnesi H. 1943 - Contribution a'l'etude des Russules de la flore francaise- 11 . Bulletin de la Societe Mycologique de France 49, 61-71.

Romagnesi H. 1945. Contribution a'p'etude des Russules de la flore francaise-III. Bulletin de la Societe Mycologique de France 61, 22-37.

Romagnesi H. 1967 - Les Russules d' Europe et d'Afrique du nord. Bordas, Paris. 998 pp.

Romagnesi H. 1972 - Sur deux Russules nouvelles de la Moitie sud de la france. Bulletin de la Societe Mycologique de France 88, 29-33.

Roze EM. 1876 - Essai Dune Nouvelle Clssification Des Agaricinees. Bulletin de la Societe Mycologique de France $23,51$.

Saini SS, Atri NS. 1981 - Russula foetens (Pers.) Fr.-A new record for India. Current Science 10, 460-461.

Saini SS, Atri NS. 1982a - North Indian Agaricales - I. Indian Phytopathology 35, 265-272.

Saini SS, Atri NS. 1982b - North Indian Agaricales-III. Society Indian National Science Academy 48B, 453-457.

Saini SS, Atri NS. 1984 - Studies on the North-West Himalayan Russulaceae. Geobios New Reports 3, 4-6.

Saini SS, Atri NS. 1989a - North Indian Agaricales-IX section Ingratae Quel. of Russula Pers. Kavaka 17, 21-27.

Saini SS, Atri NS. 1989b. North Indian Agaricales XI-Section Russula Pers. in India, Indian Journal of Mycology and Plant Pathology 19, 44-49.

Saini SS, Atri NS. 1990 - Two noteworthy taxa of Lactarius Pers. From India. Journal of the Indian Botanical Society 69, 475-476.

Saini SS, Atri NS. 1993. Studies on genus Lactarius from India. Indian Phytopathology 46, 360-364.

Saini SS, Atri NS, Bhupal MS. 1988 - North Indian Agaricales-V. Indian Phytopathology 41, 622-625.
Saini SS, Atri NS, Saini MK. 1989 - North Indian Agaricales VI. Journal of the Indian Botanical Society 68, 205-208.

Sarnari M. 1998. Monografia illustrate Del Genere Russula in Europa. Tomo Primo Italia. $799 \mathrm{pp}$.

Sarwal BM. 1984. Taxonomic studies on Indian Agarics II. Indian Phytopathology 37, 228-233.

Sathe AV, Daniel J. 1980. Agaricales (Mushrooms) of Kerala State. M. A. C. S. Monogr. 1, Part III, 1-108.

Schaeffer J. 1934 - Russula-Monographie. Annale Mycologici 32, 141-243.

Shaffer RL. 1962 - The subsection Compactae of Russula. Brittonia 14, 254-284.

Shaffer RL. 1964 - The subsection Clactarioideae of Russula. Mycologia 56, 202-231.

Shaffer RL. 1970a - Notes on the subsection Crassotunicatinae and other species of Russula. Lloydia 33, 49-96.

Shaffer RL. 1970b - Cuticular terminology on Russula. Brittonia 22, 230-239.

Shaffer RL. 1972 - North American Russulas of the subsection Foententinae. Mycologia 64, 1008-1053.

Shajahan M, Samajpati N. 1995 Ectomycorrhizal fungi of Shorea robusta G. G. from West Bengal. Indian Journal of Mycological Research 33, 105-117.

Sharma AD, Munjal RL, Seth PK. 1978 Some fleshy fungi from Himachal Pradesh-III. Indian Journal of Mushroom 4(2), 27-29.

Sharma JR, Das K. 2001 - Mycological trip of Pindari Glacier. Indian Journal of Forestry 24: 239 - 244.

Sharma JR, Das K. 2002 - New records of Russulaceae from India. Phytotaxonomy 2, 11-15.

Sharma JR, Das K. 2003 - New and interesting species of Lactarius from India. Mycotaxon 88, 377-385.

Sharma JR, Das K, Kukreti S. 2005 - Two new records of fleshy fungi from India. Indian Journal of Forestry 28, 78-80.

Singer R. 1926. Monographie der Gathung Russula Mit 1 Taf. Hedwigia 66, $163-$ 260. 
Singer R. 1928a. Drei settene all Berhalh Deutoschlands heimische Russulae. Zeitschrift für Pilzkunde 7, 42-44.

Singer R. 1928b. Neue Mitteilungen uber die Gattung Russula. Hedwigia 68, 191201.

Singer R. 1975. The Agaricales in Modern Taxonomy. 3rd ed. Vaduz J. Cramer. Germany.

Singer R. 1986. The Agaricales in Modern Taxonomy. Bishen Singh Mahendrapal Singh, Dehradun, India. 981 pp. 88 plates.

Smith AH, Hesler LR. 1962. Studies on Lactarius-III. The North American species of section Plinthogali. Brittonia 14, 369-440.

Stubbe D, Nuytinck J, Verbeken A. 2010. Critical assessment of the Lactarius gerardii species complex (Russulales). Fungal Biology 114, 271-283.

Stubbe D, Le HT, Wang XH, Nuytinck J, Van de Putte K, Verbeken A. 2011 - The Australasian species of Lactarius subgenus Gerardii (Russulales). Fungal Diversity 58, 141-167.

Van de Putte K, De Kesel A, Nuytinck J, Verbeken A. 2009 - A new Lactarius species from Togo with an isolated phylogenetic position. Cryptogamie Mycologie 30, 1-6.

Van de Putte K, Nuytinck J, Stubbe D, Le HT, Verbeken A. 2010 - Lactarius volemus sensu lato (Russulales) from northern Thailand: morphological and phylogenetical species concepts explored. Fungal Diversity 45, 99-130.

Verbeken A. 1998a. Studies in tropical African Lactarius species. 5. A synopsis of the subgenus Lactifluus (Burl.) Hesler \& A. H. Sm. emend. Mycotaxon 66, 363386.

Verbeken A. 1998b - Studies in tropical African Lactarius species. 6. A synopsis of the subgenus Lactariopsis (Henn.) R. Heim emend. Mycotaxon 66, 387-418.

Verbeken A, Horak E. 2000 - Lactarius (Basidiomycota) in Papua New Guinea. 2. Species in tropical montane rainforests. Australian Systematic Botany 13, 649-707.

Verbeken A, Walleyn R, Sharp C, Buyck B. 2000 - Studies in tropical African Lactarius species 9. Records from Zimbabwe. Systematics and Geography of P1ants. 70, 181-215.

Vrinda KB, Pradeep CK, Abraham TK. 1997 A new species of Russula from Kerala, India. Mycotaxon 62, 87-96.

Wang, Xiang Hua. 2007 - Type studies of Lactarius sp. published from China. Mycologia 99, 253-268.

Wang, X-Hu, Hashiya M, Verbeken A. 2006 Lactarius ochrogalactus, a new species of genus Lactarius (Russulaceae, Russulales) with yellowish brown latex. Mycoscience 47, 232-234.

Watling R, Gregory NM. 1980 - Larger fungi from Kashmir. Nova Hedwigia 32, 493-547. 